\title{
A Fractional Order Control Strategy for LFC via Big Bang Big Crunch \& Grey Wolf Optimization Algorithms
}

\author{
Dola Gobinda Padhan $^{1 *}$, Syed Sarfaraz Nawaz ${ }^{2}$, and Padmanabhuni Ravikanth ${ }^{3}$ \\ ${ }^{1}$ EEE Department, Gokaraju Rangaraju Institute of Engineering and Technology, Hyderabad, India
}

\begin{abstract}
The Grey Wolf Optimization (GWO) is well known meta-heuristic algorithm and has been previously used for optimization of various conventional PID and FOPID controllers. This paper deals with the application of Grey Wolf Optimizer (GWO) algorithm and internal model control (IMC) for optimization of fractional order PID (FOPID) controller parameters to the load disturbance of system. This is applicable for one (or) single area non reheated electrical system. The simulation results are compared with the non re-heated Big Bang Big Crunch (BBBC) optimization outputs. In this paper, BBBC optimization has the two bounding cases (lower \& upper), they are before and after the perturbation cases. Also it is observed that in case of the BBBC output responses, the settling time value of load frequency is more when compared with the GWO. From the simulation results it is concluded that GWO out performs as compared with BBBC as it produces less error and settling time.
\end{abstract}

\section{Introduction}

The executed outputs of major electrical system is under goes degradation in power quality obtain because of the load changes in the electrical power system [1]. It reflects the variation of the electric power flow between in one line to nearly connected another lines of the system as well as frequency of particular system area. The problem tells us to need of frequency controlling device to resolve the variations in the system load, output values uncertainties and also control to the unwanted electric energy flowing among interconnected electrical system areas. These frequency controlling device of the system load useful for the minimization of load variation issue, robustness issues in the power system [2] Different newest controlled techniques are applied, they are optimal control, sliding mode control, PID control, internal controlled scheme, etc. all these methods reduce the variation in the electric system load values [3-6]. Now, present day's method of minor calculus method have proof of most successful in electric system world. In the FOPID controlling device provides extra benefits in the designing electric power system. They are gives the five controlled values they are $k_{P}, k_{I}, k_{D}, \lambda, \mu$ in place of three parameter values.

\section{Overview of LFC}

Load frequency control is nothing but suddenly load changes in the particular area when abnormal conditions occur in the system.

\footnotetext{
*Corresponding author: dgobinda@gmail.com
}

The controlled device maintaining is most important to the electrical system. In previous years most of theories explained about load frequency control in the electrical system. Present column electric factories are changed to transform to the redesigning manner[4]. The load frequency controlled issue in the electric power system needs the not only frequency variation of every area compulsory regain their original point but also regain the tie-line energy flow need to regain their pre-defined values. If the electric system values are changes then its re-construction is difficult to the entire electric system when the system is under processing by using PID model controllers.

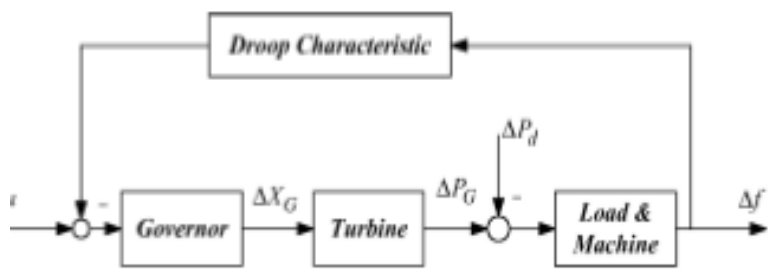

Fig.1. One area electric power system.

In this paper we are controlling such problems by designing of FOPID for better output frequency responses.

\subsection{FOPID Controller}

In present days, the fractional order controller has been very common for various real-world application problems in each field of engineering and also industrial applications. The first FOPID controllers as the command-rousted order system it is a non- entire (CRONE) controller. He implemented this 
controller in different field of control application. The success of FOPID controller is due to its extra degree of freedom which provides additional support to maintain a proper balance between the time-domain specifications of the system. Moreover, performance of FOPID controller is also proved better for many other practical systems.

$$
c(s)=K_{p}+\frac{K_{i}}{s^{\lambda}}+K_{d} \cdot S^{\mu}
$$

Above equation tells where $k_{P}, k_{I}, k_{D}, \lambda, \mu$ are five tuning parameters values of FO-PID. By using FOPID controller we can found the five parameters of the single-area electric power system for better load frequency controlling device via grey wolf algorithm.

\section{Statements of problem}

The electric power system is a major sector system having the difficultly in unlinear dynamics in the nature. Whatever, for small changes in the load variation, then it reflects the variations in the linear model of the system, liberalized in the operated point. The multi- level electric power system of LFC designing having a governor $G_{g}(s)$ un re-heated turbine $G_{t}(s)$, load \& machine device $G_{p}(s)$, drooping characteristics of the system. Each and every component values of system dynamics are listed below:

$$
\begin{aligned}
G_{g}(s) & =\frac{1+0}{1\left(1+T_{g} s\right)} \\
G_{t}(s) & =\frac{1+0}{1+T_{t} s} \\
G_{p}(s) & =\frac{K}{1+T_{p} s}
\end{aligned}
$$

In the table 1 shows the nomenclature of used symbols. Characterization of system model is explained by the given below transfer function:

$$
\Delta F(s)=T_{u}(s) \Delta u(s)+T_{d}(s) \Delta P_{d}(s)
$$

Above formula tells us to LFC is a firstly disturbed rejected issue in the system, and our major work is to develop robusted FOPID controlling device of a single area electric power system. By using this controller we want to minimize the load variations on the $\Delta F$ is minimum value and by using below formula design of FOPID controlled device.

$$
c(s)=k_{P}+\frac{K_{i}}{s^{\lambda}}+K_{d} \cdot S^{\mu}
$$

Here are $k_{P}, k_{I}, k_{D}$ denotes as proportional gain, integral gain, the derivative gain respectively. $\lambda, \mu$ denotes the fractional integral, derivative terms respectviely .

\section{Proposed approach of the GWO- FOPID}

The proposed method is divided to mainly two modes of operation, in the mode 1 tells us to the designing of the PIDIMC controller of the electrical power system. In mode 2 we utilizes the GWO algorithm to develop the optimal FOPID controlled device by helpful of PID parameter values founded in the mode1 it tells us bounding solutions area.

The route of developed calculations of an executed method is given below.

\subsection{Internal model control method}

From (1)-(4) tells us to the single area electrical power $3^{\text {rd }}$ order system. This $3^{\text {rd }}$ system can be reduced to the $2^{\text {nd }}$ order system before the IMC approachment. In this work this order decrement is via GWO algorithm. Because it is easy to knowing the concept and also this $2^{\text {nd }}$ order method reflects the original system value. Reduced order method formula is given below.

$$
G_{R}(S)=\frac{a_{0}+a_{1} s}{b_{0} s^{2}+b_{1} s+b_{2}}
$$

Where $a_{j}, \mathrm{j}=0,1 \& b_{i}, \mathrm{i}=0,1,2$

Coefficients to $\mathrm{S}, \& b_{i}>1$.

The load frequency variation control problems we identified the decreased order method occurred via GWO is gives us to the non -minimum phase value system for that we need the $a_{1} \leq 0 \& a_{1} \geq 0$.

The exact process is need to apply for the GWO algorithm of the system. Then from (6) can be re-written as

$$
\begin{aligned}
& G_{R}(S)=\frac{a_{0}\left(1+a_{2} s\right)}{b_{0} s^{2}+b_{1} s+b_{2}} \\
& \text { Where } \quad a_{2}=\frac{a_{1}}{a_{0}}<1
\end{aligned}
$$

After occurring of decreased order method then we execute the IMC method to LF control issue.

.Diagram. 2 shows block diagram of the basic IMC control.

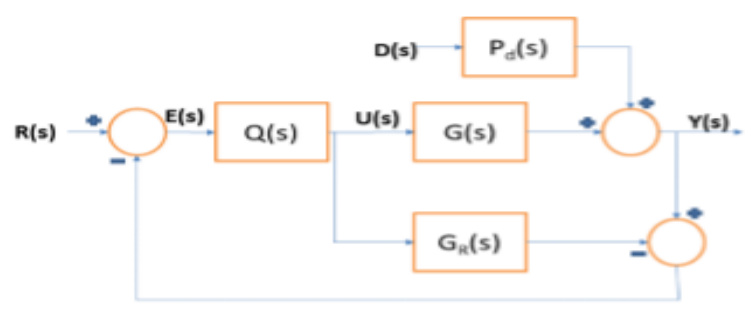

Fig.2. Basic IMC control scheme.

The plant model is factorized as

$$
G_{R}(s)=G_{R-}(s) G_{R+}(s)
$$

Where $G_{R_{-}}(s)$ and $G_{R+}(s)$ represent the minimum and non- minimum phase parts respectively.

$$
\begin{aligned}
& G_{R-}(s)=\frac{a_{0}}{b_{0} s^{2}+b_{1} s+b_{2}} \\
& G_{R+}(s)=\left(1+a_{2} s\right)
\end{aligned}
$$

Further next step formula is written below 


$$
f(S)=\frac{1}{(1+\delta s)^{K}}
$$

Here, $\delta$ is intuitively modulated value. $\mathrm{K}$ represents the IMC controlled device. Here $\mathrm{k}=1$.

End of the operating work IMC controlled device gives the below formula

$$
Q(s)=f(S) G_{R-}^{-1}(s)=\frac{b_{0} s^{2}+b_{1} s+b_{2}}{a_{0}(1+\delta s)}
$$
below

PID controller formula in the feed -back mode is writes

$$
C_{I M C}(s)=\frac{Q_{s}}{1-G_{R}(s) Q(s)}
$$

The replacement of these values in $G_{R}(s) \& \mathrm{Q}(\mathrm{s})$ through equations (7),(12).

$$
C_{I M C}(s)=\frac{b_{1}}{a_{0} \delta-a_{1}}+\frac{b_{2}}{a_{0} \delta-a_{1}} \frac{1}{s}+\frac{b_{0}}{a_{0} \delta-a_{1}} s
$$

Formula (16) is again wrote by

$$
C_{I M C}(s)=k_{P}+k_{i} \frac{1}{s}+K_{d} s
$$

Where $K_{P}=\frac{b_{1}}{a_{0} \delta-a_{1}}, K_{i}=\frac{b_{2}}{a_{0} \delta-a_{1}} \& K_{d}=\frac{b_{0}}{a_{0} \delta-a_{1}}$.

Now finally we occurred values to the PID-IMC control scheme. Then further step is the occurred values is useful to found the solution of FO-PID via GWO algorithm technique.

\subsection{BB-BC Algorithm}

From This optimization technique, mainly it has two phases BB phase \& BC phase. BB phase will tells us solution of the population evaluated in irregularly in a distributed area.

$\mathrm{BC}$ phase tells us single representative solution is nothing but center of mass solution.

This optimization technique starts real-power distributed in the atmosphere. This BB phase reflects the searching area procedure and $\mathrm{BC}$ phase represents the better outputs of the system.

This procedure for BB-BC algorithm to find solution of unwanted load frequency in one area non re-heated electric power system.

Step 1: initialize this BBBC parameters like $N, \psi$, matrix $\&$ iteration and using the below formulas we can found the lower bound \& upper bound parameters.

Where $k_{p}, k_{i}, k_{d}$ are PID parameter values from equation (15). $\lambda$ and $\mu \in(0,2)$. If $\lambda, \mu \geq 0$,

$$
\begin{aligned}
& L=\left[\begin{array}{ll}
p^{-1} k_{p}, p^{-1} k_{i}, & p^{-1} k_{d}, 0,0
\end{array}\right] \\
& \mathrm{U}=\left[\begin{array}{ll}
p k_{p}, p k_{i}, p k_{d}, 2,2
\end{array}\right]
\end{aligned}
$$

Step 2: it generates $\mathrm{N}$ candidate solution is called Big Bang Phase (BBP).

$$
x_{i}=L+(U-L) o R \quad i=1,2, \ldots \ldots . N
$$

Where $\quad x_{i}=$ vector, $i=$ candidate iteration

Step 3 \& step $4:$ In the $3^{\text {rd }}$ stage tells us the optimized mathematical matrix of the number of students results, and in the $4^{\text {th }}$ stage tells us the order of the fitnessed results with their magnitude values.

Step 5: in this method tells us to number of newly presented candidates solution in searching area according to the origin mass developed in the last stage. By useful formula written below

$$
x_{i}^{i t e r+1}=C^{i t e r}+\frac{r_{i} \varphi(U-L)}{i t e r+1}
$$

Step 6: In this stage fitness matrix set values of newly generated candidate solutions, and repeated the process until final value occurred.

The BBBC algorithm gives large settling time value when controlling load frequency in non re-heated single area power system when control parameter values changes to $+50 \%$ means upper bound \& $-50 \%$ lower bound parameters. This problem is solved by using grey wolf optimization control scheme to control load frequency disturbance problem by using below procedure.

\subsection{Grey wolf (GWO) optimization algoritham}

GWO is a one of the optimized method to development of proportional, integrative, derivative controller. The GWO algorithm is useful to the checking the behavior of wolfs group. According to analysis of making between potential solution of the problem, number of wolfs chasing the prey.

Below diagram having the stages of wolfs $\mathrm{a} / \mathrm{c}$ to the hunting process.

\subsubsection{GWO Levels}

\section{Hunting levels of wolfs.}

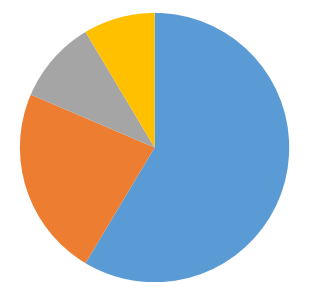

\section{Alphas ( $\alpha)$ :}

It is gives the better solution when compared to remaining wolfs. These are the head of the total wolfs.

2. Betas $(\boldsymbol{\beta})$ : these are gives the $2^{\text {nd }}$ better population solution of the total wolfs. It is subordinate the alphas.

3. Delta (ס): these are represents third order wolfs and subordinates to the beta.

4. Omegas $(\omega)$ : these are subordinates to all the rest of population solutions.

\section{A. MATHEMATICAL MODEL}

In the modeling, the GWO Algorithm resembles the leadership hierarchy and hunted system of the wolves.

\section{SOCIAL POINT VIEW}


The model of social view of hierarchy is carries the alpha $(\alpha)$ gives us best solution follows by beta $(\beta) \&$ delta $(\delta) \&$ omega $(\omega)$.

\section{EN-CIRCLING PREY}

The Grey wolves rounded the prey and chases them up to the prey stops at un movement stage means, prey stops stand still condition. The given below formulas tells the behavior of the wolves.

$$
\begin{aligned}
& \vec{D}=\overrightarrow{\mid C} \cdot \overrightarrow{x_{P}}(t)-\vec{x}(t) \mid \\
& \vec{x}(t+1)=\left|\overrightarrow{x_{P}}(t)-\vec{A} \cdot \vec{D}\right|
\end{aligned}
$$

Where t means iteration number, $\vec{A} \& \vec{c}$ means co-efficient vectors, $\vec{x}_{p}$ meaning of the position vector of the prey $\& \vec{x}$ stands for position vector of the Grey Wolfs. $\vec{A} \& \vec{c}$ vector calculation as given below,

$$
\vec{A}=2\left(\vec{a} \cdot \overrightarrow{r_{1}}-\vec{a}\right) \quad \& \quad \vec{C}=2 \cdot \overrightarrow{r_{2}}(1)
$$

Here contents of $\vec{a}$ are linearly dropped from 2 to 0 in the course of iteration \& $\overrightarrow{r_{1}}, \overrightarrow{r_{2}}$ are randomly developed vectors in $[0,1]$. From above mentioned equations, the Grey Wolf is capable of upgrading the it's position of the (X, Y) depends on the position of the prey $\left(\mathrm{X}^{*}, \mathrm{Y}^{*}\right)$.and also develops the new position is based on the current position adjustment of the values of $\vec{A} \& \vec{C}$ vectors. The movement of Grey Wolves having like squares and circles.

\section{HUNTING}

The hunting process will be handled through the alphas. Grey wolves is majorly advised by the alpha.

The following equations are useful for the grey wolf optimization for finding the load frequency problem for nonreheated power system.

$\overrightarrow{D_{\alpha 1}}=\left|\vec{C}_{1} \cdot \overrightarrow{x_{1 \alpha}}(t)-\vec{x}(t)\right|, \overrightarrow{D_{\beta 1}}=\left|\vec{C}_{2} \cdot \overrightarrow{x_{\beta 1}}(t)-\vec{x}(t)\right|$

$\overrightarrow{D_{\delta 1}}=\overrightarrow{\mid C_{3}} \cdot \overrightarrow{x_{\delta 1}}(t)-\vec{x}(t) \mid$

$\overrightarrow{x_{1}}=\overrightarrow{x_{\alpha 1}}-\overrightarrow{A_{1}} \cdot \overrightarrow{D_{\alpha 1}}, \overrightarrow{x_{2}}=\overrightarrow{x_{\beta 1}}-\overrightarrow{A_{2}} \cdot \overrightarrow{D_{\beta 1}}$

$\overrightarrow{x_{3}}=\overrightarrow{x_{\delta 1}}-\overrightarrow{A_{3}} \cdot \overrightarrow{D_{\delta 1}}, \quad \vec{x}(t+1)=\frac{\overrightarrow{x_{1}}+\overrightarrow{x_{2}}+\vec{x}}{3}$

\section{ATTACKING THE PREY}

This mode is activated after the hunting is completes when prey at stand still condition.

Below shows the flow chart diagram for grey wolf optimization diagram for finding procedure of the better frequency response in single area non-reheated turbine power system.

\section{SIMULATION STUDY}

From figure 1 we observed that the non re-heated turbine single area power ststem and drooping characteristics of the system. This simulation is developed in the Matlab software .the useful parameter values of LFC are listed below. They are $\mathrm{K}=120, T_{g}=0.081, T_{p}=21, T_{t}=0.4, \mathrm{R}=2.41$

By using the above values, the plant model in the transfer function form is written in the below

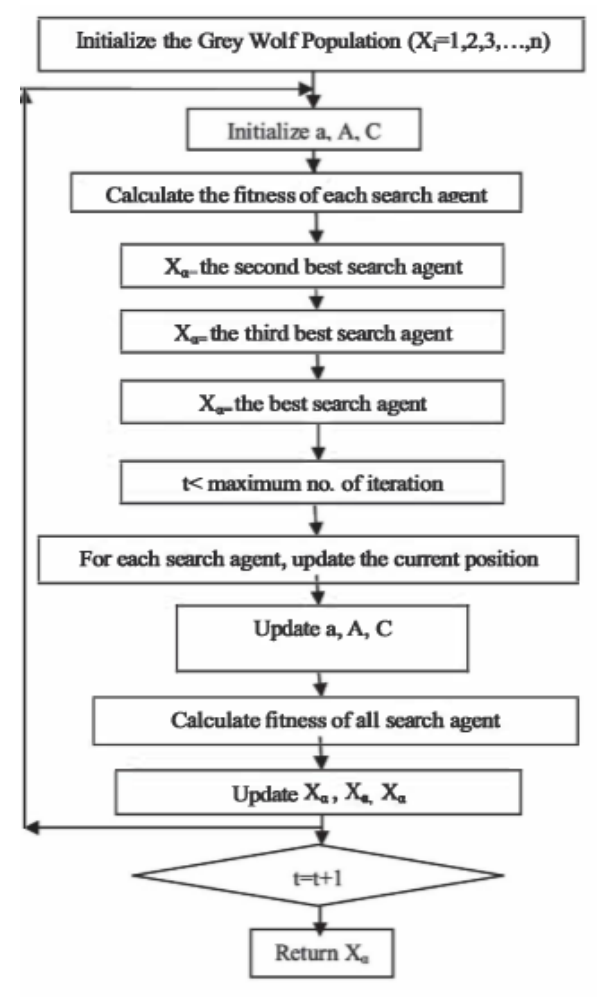

Fig.3. Flow chart for GWO algorithm

$$
G(s)=\frac{250}{s^{3}+15.88 s^{2}+42.45 s+106}
$$

The conventionalized IMC-PID control device is obtained by using the equations from (7) - (15) and these are gives the typical parameter values of the GWO for developing the FOPID controllers

In the required $K_{p}, K_{i}, K_{d}, \lambda$ and $\mu$ values are found by using formulas written in above grey wolf optimization chapter by solving through the IMC-PID equations. These equations are resolved into two degree

Equations and this equations are found transfer function values. These control parameter values are substitute in the PID control diagram designed in the MATLAB software.

In the GWO optimization technique is gives better frequency response settling time value. It is also reduce the IAE, ITAE, STD errors in the single area non-reheated power system when compared with the BBBC optimization technique.

GWO is also gives the better response values after perturbation case means after changing the control parameter values of both lower bound and upper bound cases.

Below shows the MATLAB designed diagrams of BBBC \& GWO and their respective output waveforms and performance table values and comparison values. 


\section{Block diagrams \& simulation results}

Block diagram of FOPID-BBBC \& GWO

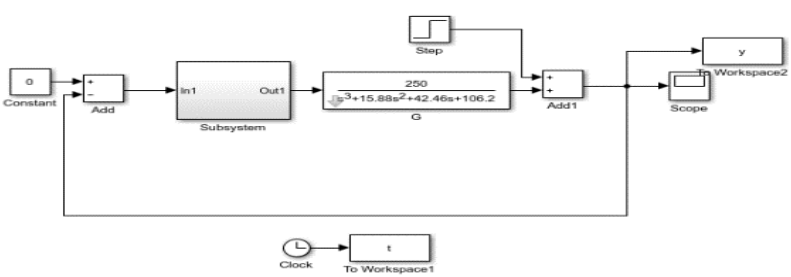

Fig.4. Block diagram of FOPID-BBBC

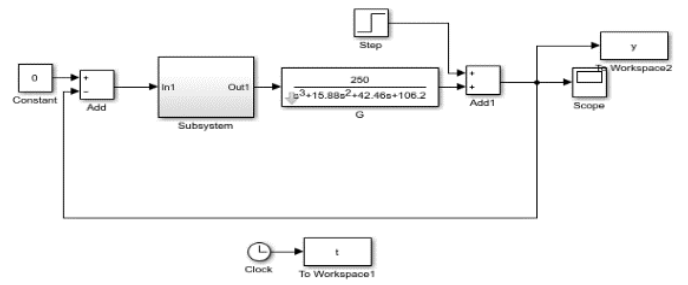

Fig.5. Block diagram of FOPID-GWO

Below waveforms tells the frequency response with respect to the time of the BBBC \& GWO optimization techniques. we observed that the settling time of grey wolf optimization is gives better settling value when compared to the BBBC optimization technique.

The red line tells the GWO optimization line \& sky blue line tells the $\mathrm{BBBC}$ optimization technique. The respective frequency response outputs of $\mathrm{BBBC}$, GWO lower and upper bound combination are shown in Fig.7.

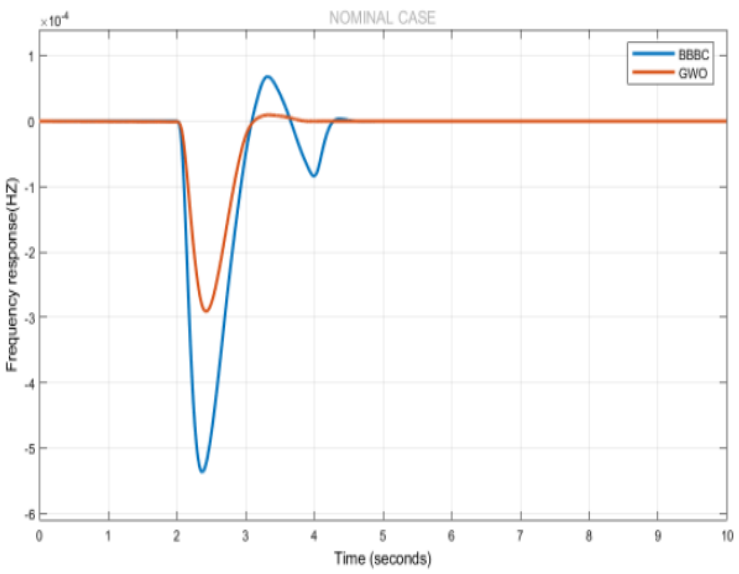

Fig. 6. Frequency response of both $\mathrm{BBBC} \& \mathrm{GWO}$ nominal case

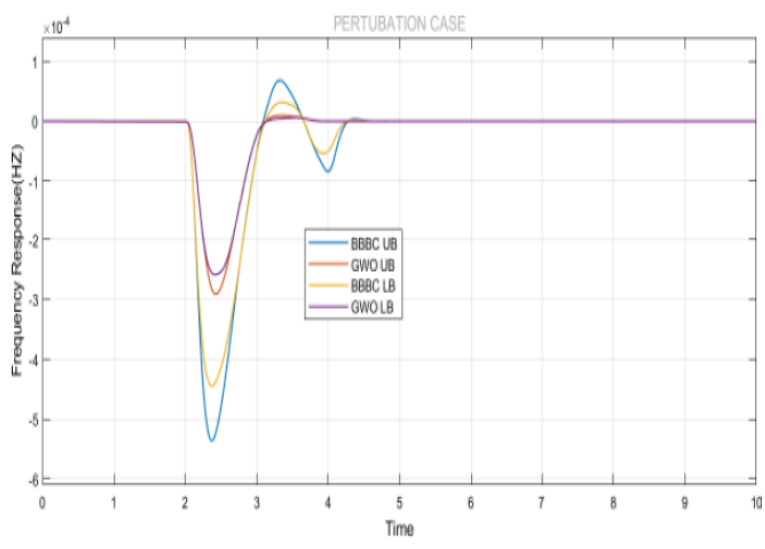

Fig.7. Frequency response Perturbation case

Table 1. FOPID parameters obtained via GWO

\begin{tabular}{llllll}
\hline Parameter & Min & Max & Mean & Var & STD \\
\hline$K_{p}$ & 0.20197940 & 18.65114684 & 5.79760800 & $1.2820153 e^{+01}$ & $3.58052421 e^{+00}$ \\
$K_{i}$ & 0.07790634 & 9.54262745 & 3.10755504 & $3.70923323 e^{+00}$ & $1.92593697 e^{+00}$ \\
$K_{d}$ & 0.59223245 & 7.79063393 & 2.03142790 & $1.72152149 e^{+00}$ & $1.31206764 e^{+00}$ \\
$\lambda$ & 0.00000000 & 1.54639499 & 0.52338854 & $8.25679308 e^{-02}$ & $2.87346360 e^{-01}$ \\
$\mu$ & 0.00000000 & 1.29445383 & 0.33309164 & $3.81049823 e^{-02}$ & $1.95204975 e^{-01}$ \\
$t_{s}$ & 3.61807887 & 3.61807887 & 3.61807887 & $4.43734259 e^{-29}$ & $6.66133815 e^{-15}$ \\
$t_{r}$ & $0.00000001 e^{+00}$ & $0.00000020 e^{+00}$ & $0.00000400 e^{+00}$ & $0.00000510 e^{+00}$ & $0.00066000 e^{+00}$ \\
ISE & $3.47411623 e^{-08}$ & $3.47411623 e^{-08}$ & $3.47411623 e^{-08}$ & $2.14573827 e^{-45}$ & $4.63221143 e^{-23}$ \\
IAE & $1.65684707 e^{-04}$ & $1.65684707 e^{-04}$ & $1.65684707 e^{-04}$ & $1.05794492 e^{-37}$ & $3.25260652 e^{-19}$ \\
ITAE & $4.17166358 e^{-04}$ & $4.17166358 e^{-04}$ & $4.17166358 e^{-04}$ & $7.52316385 e^{-37}$ & $8.67361738 e^{-19}$ \\
\hline
\end{tabular}

Best solution is $K_{p}=5.49772066, K_{i}=3.13332847, \quad K_{d}=1.77083909, \lambda=0.47069739, \& \mu=0.29249624$ 
Table 2. Performance indices for BBBC nominal and perturbation cases

\begin{tabular}{|c|c|c|c|c|c|c|}
\hline Design method & ISE & IAE & ITAE & Nominal case: ISE & IAE & ITAE \\
\hline LOWER BOUND & $2.7105 e^{-07}$ & $6.8817 e^{-04}$ & $2.6528 e^{-03}$ & $2.7105 e^{-07}$ & $6.8817 e^{-04}$ & $2.6528 e^{-03}$ \\
\hline UPPER BOUND & $2.7111 e^{-07}$ & $6.8813 e^{-04}$ & $2.6529 e^{-03}$ & $1.5842 e^{-07}$ & $6.1078 e^{-04}$ & $2.4937 e^{-03}$ \\
\hline
\end{tabular}

Table 3. Performance indices for GWO nominal and perturbation cases

\begin{tabular}{llllll}
\hline Design method & ISE & IAE & IATE & Nominal case: ISE & IAE \\
\hline LOWER BOUND & $1.73646733 e^{+03}$ & $4.69624526 e^{+01}$ & $2.10555524 e^{+01}$ & $1.7105 e^{-07}$ & $5.8817 e^{-04}$ \\
UPPER BOUND & $1.76637993 e^{+01}$ & $5.04870979 e^{+01}$ & $3.15544362 e^{+01}$ & $1.8842 e^{-07}$ & $5.1078 e^{-04}$ \\
\hline
\end{tabular}

\section{Conclusion}

Finally this work is done the development of an optimized FOPID controller to the single area with the non -reheated turbine electrical power system via GWO optimized algorithm through the IMC scheme. The proposed method is have extremely fast disturbance rejection capability to the load frequency disturbance, when compared with the BBBC optimization technique. GWO optimization technique gives better parameter values when before and after the perturbation cases. And also lower and upper bound cases of $\mathrm{BBBC}$ and GWO frequency responses also found in this paper.

This process may be applicable to the power system with re-heated turbine and hydro turbines.

\section{References}

1. Shivam Jain, Yogesh .V, Design of fractional PID for Load frequency control through the Internal model control and Big bang Big crunch optimization., IFAC papers On Line, Volume 51, Issue 4, pp. 610-615, 2018

2. R. K. Cavin, M. C. Budge and P. Rasmussen, An Optimal Linear Systems Approach to Load-Frequency Control, IEEE Transactions on Power Apparatus and Systems, vol. PAS-90, no. 6, pp. 2472-2482, Nov. 1971,

3. Muro C, Esscobedo R, Spector L, and Coppinger RP, Wolf-pack (Cannis lupus) Hunting strategies Emerging from simple rules in computational simulations, Behavioural Processes, Volume 88, Issue 3, pp. 192-197, 2011.

4. D. G. Padhan and S. Majhi, A New Control Scheme for PID Load Frequency Controller of Single-area and Multiarea Power Systems, ISA Transactions, vol-52, pp 242 251, 2013.

5. S. Mirjalili, S M. Mirjalili, and A Lewis, "Grey wolf optimizer," advances in engineering software, Volume 69, pp 46-61, 2014.

6. Biradar, S., Hote, Y.V., and Saxena, S., Reduction ordered modeling of linear time in-variant systems using BB-BC optimization and \& time moment matching method. Applicable Mathematically Modeling, Vol-40, No 15, 7225-7244, 2016 